\title{
GENE IMPRINTING: ENGRAVING THE PATHOGENESIS OF HERIDETARY DISEASES
}

\author{
${ }^{1}$ Sandeep Satapathy, ${ }^{2}$ Roshan Kumar Singh and ${ }^{1}$ Harikrishnan Rajendran \\ ${ }^{1}$ Departement of Biological Sciences, Indian Institute of Science Education and Research, Bhopal, India \\ ${ }^{2}$ Departement of Zoology, University of Delhi, New Delhi, India
}

Received 2014-01-11; Revised 2014-01-21; Accepted 2014-02-12

\begin{abstract}
Gene imprinting has conduited the scope of our understanding of phenotypic expression and its corelation with constituent genotype. It is an epigenetic process that involves DNA methylation and histone modulation to attain monoallelic gene expression without altering the genetic sequences. A distinctive model of non-mendelian genetics, imprinting extends the control over expression of traits and selection of the allele that would direct the same, in a manner decided by the parent of origin. The constitutive existence of this imprinting even after gametogenesis, throughout the somatic development extends a clue for its regulatory hold on several heridetary traits. Several heridetary diseases like Cancers, RussellSilver syndrome, Beckwith-Wiedemann syndrome, Prader-Willi and Angelman Syndromes and Neurodegenration have shown to be a subsequent error in the genomic impriting process. So, understanding these epigenetic regulations can be a therapeutic strategy for disease modelling and especially targeting their patterns of heridetary inheritance.
\end{abstract}

Keywords: Gene Silencing, Cancers, Neurodegenration, PEGs, MEGs, Heridetary, Russell-Silver Syndrome, Beckwith-Wiedemann Syndrome, Prader-Willi and Angelman Syndromes, Uniparental Disomy (UPD)

\section{INTRODUCTION}

The mammalian chromosome pair is sourced equally from paternally and maternally inherited allels, which are equally probable to be expressed. Thus the expression of the chromosomes and downstream functionalities associated with them are uniquely unbiased and situationally selected varying from system to sytem. Adding to this, we have examples in which the monoallelic gene expression ependenson which parent they are inherited from. Thus, PEGSs or paternally expressed genes are allowed only to be expressed when they are inherited from the father and are silenced for the one which is maternally inherited. In contrast the MEGs or maternally expressed genes follow a pattern in reverse. However, determining its temporal occurence, has shown that the process of silencing (or genomic imprinting) of one allele occurs during the stage of gametic development and subsequently this pattern is held steady in the stage of fertilization andeven thereafter throughout somatic development (Fig. 1) (Monk and Surani, 1990). The sole mechanism of this genetic imprinting are epigenetic modifications, like DNA methylation at the Imprinting Control Regions (ICRs) present on the genes. One of the most intruiging feature of this expressional system is that, each ICR naturally defines the parental expression of numerousadjacent genes (Ohlsoon et al., 1995).

The classic example of genetic imprinting is IGF2, a growth factor gene normally expressed when inherited from the father, but mutedwhen inherited from the mother. Genes like IGF2 are hushed by mammalian mothers reason being that only the mothers have to bear the cost of gestation and giving birth to a offspring. The paternal parent on the other hand has nothing to its loss, thus gets the maximum benefit of the offspring, but has no cost to bear, thus activating his part of copy in the offspring.

Corresponding Author: Sandeep Satapathy, Departement of Biological Sciences, Indian Institute of Science Education and Research, Bhopal, India Tel: +91-7898664419 


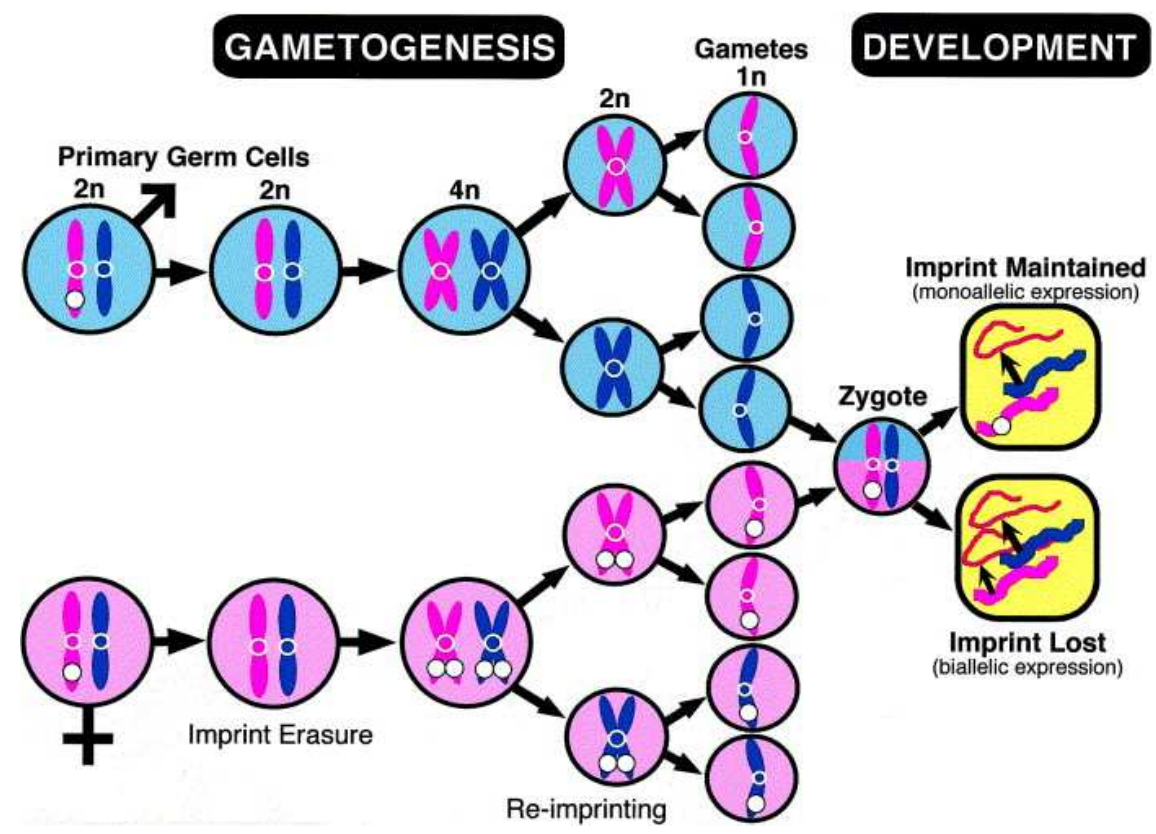

Fig. 1. Source: http://www.sciencedirect.com/science/article/pii/S1383574298000180

\subsection{Implications of Genetic Imprints}

Genetic imprinting provides a regular growth pattern,with high order of precision over the expression of the genes; dictated by the time,requirement and favourability. This expression pattern conduits the organism's struggle for a normal and healthy life (Munusamy et al., 2013). However, environmental stressors or fluctuations can affect thecontrolled regulation or induce random genetic modifications leading to abnormality and subsequent emergence of cellular catastrophe.

\subsection{Target for Heridetary Diseases}

The molecular mechanism of genetic imprinting has led to the discovery of its hereditary flow and the precision of this passage has explained the systemic growth and stability. Some of the noteworthy instances of these hereditary target identification, can be summarized as:

- Pronuclear transplantation and parthenogenic experiments in mice (Surani, 1995; Strain et al., 1995)

- Phenotypes of triploids in humans

- Expression of uniparental chromosomal disomies in humans and mice (Ledbetter and Engel, 1995)

- Expression of chromosomal deletions in humans

- Expression of transgenic material in mice
- Expression of specific genes in humans and mice, in particular those related to growth, development and behavior

Some of the excellent literature studies further escalate our understanding of this subject matter (Hall, 1990; Ledbetter and Engel, 1995; Sapienza, 1994; Nicholls, 1994). Studies on genetic imprinting and its hereditary flow dynamics, has been primary focus of researchers trying to understand the genetic regulation and expression from an "imprinting" point of view. This section concentrates on addressing the clinical importance of genomic imprinting.

Studies of genetic imprinting in mice (at early stage of development) have proved that the differential expression following either maternal or paternal inheritance influence attributes like growth, behavior, placental size and survival (Cattanach et al., 1995). These factors are regulated by a very accurate and predictable influence of genetic imprinting on the gene expression depending on their parental origin (Barlow, 1994). Although each organism has two copies of the same gene, however the principle of imprinting dictates functionality. This sex biased expression gets nullified in successive generations, when that offspring passes the gene on to his or her child, both grandparental genes act as if they are inherited from that sex parent (Barlow, 1994). This means that genetic 
imprinting considers the sex of the parent and identifies no sex bias from the grand parent. In order to find the localization of these precise phenomena, studies have been intensely carried out in mice and most recently on chromosome 15 in humans (Tirumalai and Bagchi, 2013). These studies have identified spatial regions of the chromosome instead of single or a group of genes as primary focus. Early work involving imprinting effects in transgenic mice also showed that methylation acted as molecular switches for expressional patterns by turning the imprinted genes on and off. In human cases, the two importance areas of focus are:

- Chromosome 15q11-13-"Prader-Willi/Angelman region"

- Chromosome 11p14-“Insulin-like growth factor 2/H19 region"

Homology of these genes to that of mice has been established and further effects of parental derivation on genetic expression has been demonstrated. Adding to this has been the identification of differential replication in cell cycle (i.e., early Vs late) (Munusamy et al., 2013) and differential recombination during meiosis on the maternal compared with the paternal chromosomes (i.e., differences in crossover rates) (Neumann et al., 1995; Saal, 2002; Eggermann et al., 2009).

\subsection{Cancer Model}

The centrality of the roles played by genetic imprinting, is its involvement with cellular growth. Analyzing the precision of genetic imprinting and the associated fluctuations, the obvious understanding helps to explain the straightforward correlation of genetic imprintingand cancer. Carcinogenesis considers both the genes promoting and inhibiting cellular growth. Considering the pathway of genetic imprinting and its stage specificity, it has been proved that genes that function during embryologic and fetal growth may be the same genes involved in cancer at a later developmental time (Duncan et al., 1990; Monk et al., 2002). Several types of cancer have been identified as consequence of genetic imprinting irregularities. Examples of such abnormalities and cancer manifestation are, Wilms tumor gene and loss of heterozygosity for the retinoblastoma gene involving selective loss from maternal chromosomes 11 and 13 respectively. Some of the well-established instances of genetic imprinting in cancers are:

- Chromosome 1with a tumor suppressor locus, is involved in neuroblastoma (Glenn et al., 1996)
- Hereditary paragangliomas are exclusively inherited from the father (Adams, 2008)

- Multiple endocrine neoplasia type II b have been identified with the parent-of-origin dictated new mutations in the RET oncogene (Glenn et al., 1996) It would also appear that there is a possible role for imprinting of the RET gene during development

Pedigree examination has proved that a particular disorder shows genomic imprinting (Hall, 1990). Suspection of genomic imprinting interference arises from thehereditary pattern of disorder, whether its expression is guided by the parent of origin (Fig. 2). The major decisive factor in such a case is the sex of the parent transmitting the disorder that matters, instead of the sex of the expressing individual. The sex of the transmitting parent is statistically more accountable for making such pedigree calculations instead of the sex of the affected individual. Presence of discordance among monozygotic twins (Adams, 2008), theBeckwith-Weidemann syndrome (transmitted from a female) and paragangliomas (transmitted from a male) are few examples.

\subsection{Beckwith-Wiedemann Syndrome (BWS)}

Beckwith-Wiedemann Syndrome (BWS) (Fig. 3), characterized as an overgrowth disorder (hemihypertrophy, macroglossia and visceromegaly) usually present at birth is accompanied by a high risk of childhood cancer and certain congenital features. Its relational mapping to $11 \mathrm{p} 15$ chromosome defines the disorder. Worldwide, 1 in 12,000 newborns are found to manifest BWS (Adams, 2008). The condition may actually be more common than this estimate because some people with mild or unusual symptoms are never diagnosed.

Genomic imprinting in Beckwith-wiedemann syndrome was first reported when the pertaining maternal transmission of mutations was observed in some BWS families. Approximately about 10-20\% of BWS are susceptible to embryonic tumors, the most frequent are Wilms' tumors or nephroblastoma and adenocortical carcinoma (Viljoen and Ramesar, 1992). The rate of Wilms' tumor formation in the BWS population is 1000 -fold higher than in the normal population and these tumors often show pertaining loss of maternal 11p15 chromosome. Most BWS cases arise irregularily however, in both irregular and familial forms, a small percentage exhibits UPD at chromosome 11 p15 (Adams, 2008; Viljoen and Ramesar, 1992). In these cases, the remainder of the chromosome is biparental in inheritance, indicative of somatic mosaicism through a post fertilization mitotic recombination events. 


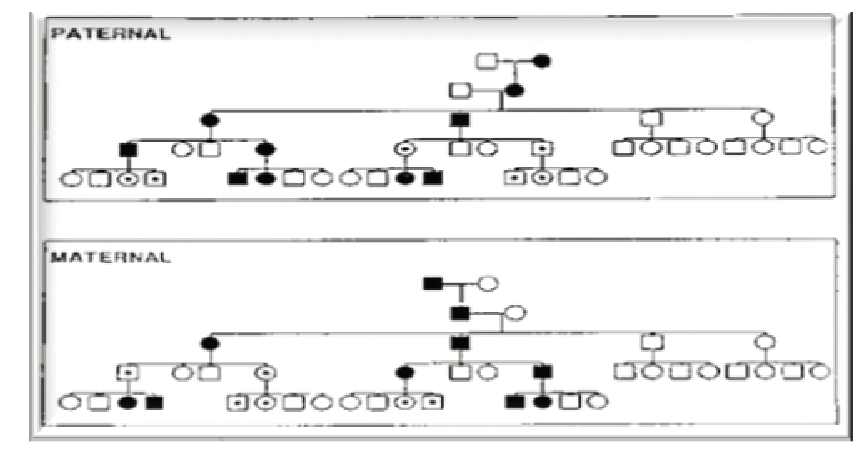

Fig. 2. Pedigree Analysis for detection of genomic impriting and disease pathogenesis (Source: J. G. Hall, Annual Review of Medicine, Vol. 48: 35-44)

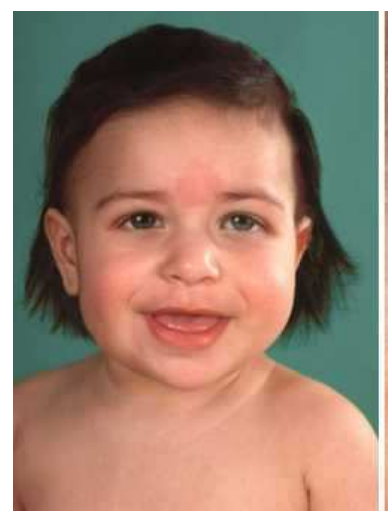

(a)

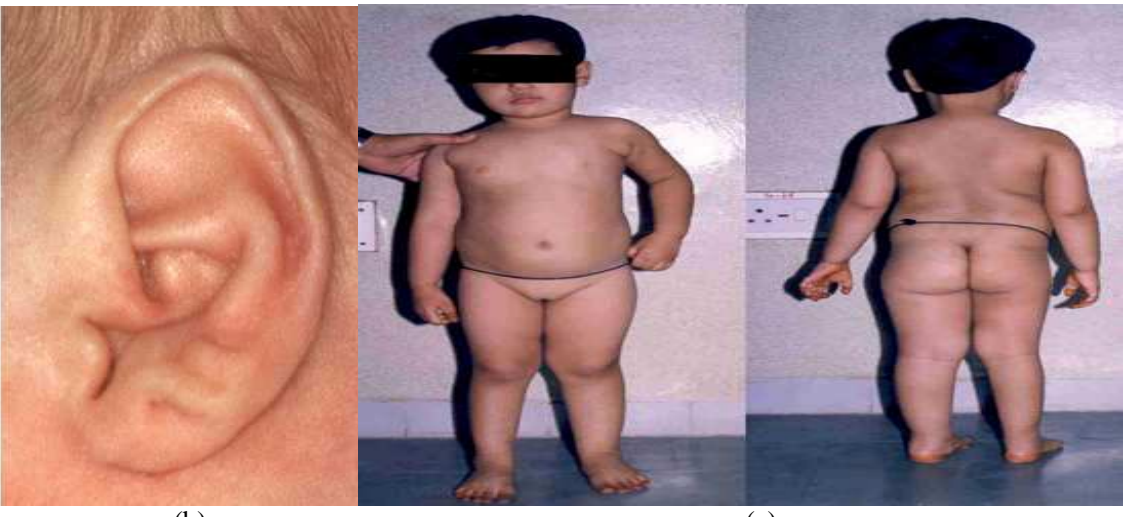

(b) (c)

Fig. 3. Patient with Beckwith-Wiedemann syndrome. (a) Macroglossia- face withenlarged tongue (b) ear-the typical earlobe creases (c) Child with isolated hemihypertrophy involving left lower limb with are association of Poland anomaly and hypoplastic nipple on left side. (Source-Atlas of Genetics and Cytogenetics in Oncology and Haematology)

The most common molecular event occurring in BWS patients is the absence of cytogenetic abnormalities in the biallelic expression of Insulin Like-Growth Factor (IGF2) due to Loss Of Imprinting (LOI) (Magenis et al., 1987; Viljoen and Ramesar, 1992). LOI at the IGF2 locus may be accompanied by the methylation or silencing of the active maternal allele of Insulin likegrowth factor/H19 (Adams, 2008). This H19dependent event is consistent with an enhancercompetition model for the co-regulation of these genes. Translocations in BWS patients may also lead to LOI at the IGF2 locus, but without loss of H19 imprinting (Reik et al., 1995). These translocations affect imprinting by disrupting a gene involved in imprint control, or by altering the function of an Imprinting Center (IC). Therefore, disruption of IGF2 imprinting in BWS may also occur via an H19independent event (Viljoen and Ramesar, 1992). The imprinted KvLQT1 gene located centromeric to IGF2 spans a common breakpoint region in BWS and has been proposed to maintain regional imprint control at 11p15.5. KvLQT1 (Reik et al., 1995) shows preferential expression from the maternal allele in most tissues examined except the heart where it is biallelically expressed (Adams, 2008; Viljoen and Ramesar, 1992). This explains why KvLQT1, responsible for the autosomal dominant cardiac arrhythmia long QT syndrome, shows no parent-of-origin effect in this disorder. The maternally expressed p57Kip2, which encodes for a cyclin-dependent kinase inhibitor, also maps to 11p15.5 (Adams, 2008).

Abnormal imprinting and epigenetic silencing of p57Kip2 is found in some individuals with BWS and mutations are present in about $5 \%$ of BWS patients (Magenis et al., 1987; Viljoen and Ramesar, 1992). To date, ten imprinted genes have been mapped to11p15.5. 
Flanking these imprinted genes are the non-imprinted NAP2 (centromeric border) and L23MRP (telomeric border) genes (Adams, 2008; Magenis et al., 1987). The syntonic region in the mouse, distal chromosome 7 , confirms the existence of an imprinting cluster at this chromosomal location. A possible explanation for the involvement of multiple genes in BWS (even if IGF2 overexpression is directly responsible for BWS) is that one or more of the adjacent genes (e.g., H19, p57Kip2, KvLQT1) are involved in the regulation of IGF2 expression (Adams, 2008). Experimental evidence supports this postulate since transgenic mice that overexpress Igf2 develop symptoms similar to BWS.

Potency of tight-binding inhibitor p57kip2 of several G1 cyclin/Cdk complexes and is a negative regulator of cell proliferation (Viljoen and Ramesar, 1992). The gene encoding p57Kip2 is located at $11 \mathrm{p} 15.5$, a region which is implicated in both sporadic (isolated instances) cancers and Beckwith-Wiedemann syndrome, Several types of childhood tumours including Wilms' tumour or nephroblastoma, adrenocortical carcinoma and rhabdomyosarcoma exhibit a specific loss of maternal $11 \mathrm{p} 15$ alleles (Magenis et al., 1987), it suggesting that genomic imprinting is involved in whole mechanism. Previously, we and other researchers observed that p57Kip2 is imprinted and that only the maternal allele which is expressed in both mice and humans (Adams, 2008):

Uniparental Disomy (UPD): A unique feature to imprinted conditions is the unusual situation in which a child inherits both copies of a chromosome from one parent and none from the other. This is known as Uniparental Disomy (UPD). Uniparental disomy usually arises due to an error in meiosis. Preece et al. (1997) Two chromosomes in either the egg or sperm cell fail to separate and both get passed to the fetus. As a result, the fetus inherits three chromosomes (trisomy) rather than two. In relatively rare situations, one of the three chromosomes is lost (termed trisomy rescue), resulting in a 'normal' two-chromosome state (disomic) after fertilization. One-third of the time, this loss will result in uniparental disomy (Henry et al., 1991).

In about $85 \%$ of cases of BSW, only one person in a family has been diagnosed with that particular instance (Adams, 2008). Other 10-15 percent people with BSW are part of families with more than one effected family member. In most of these families, the condition appears to have an autosomal dominant pattern of inheritance.15$30 \%$ of BWS cases have a mutation in the gene p57kip2 (Viljoen and Ramesar, 1992) and less than 1\% cases due to chromosome abnormality involving a region on chromosome 11 (Magenis et al., 1987).

\subsection{Russell-Silver Syndrome (RSS)}

Russell-Silver Syndrome (RSS), a growth disorder, is characterized by slow growth before and after birth. Such babies have a low birth weight and sometimes fail to grow and gain weight at the expected rate (Fig. 4). Children with RSS are thin and have poor appetites or craving and few develop low blood sugar (hypoglycemia) as a result of feeding difficulties. Phenotype of children with RSS involves a small, triangular face with distinctive facial features including a prominent forehead, a narrow chin and a small jaw whereas adults with RSS are short, the average heighted (4 feet, 11 inches-males and 4 feet, 7 inchesfemales) (Eggermann et al., 2009; Duncan et al., 1990; Kotzot et al., 1995). Russell-Silver syndrome is estimated about 1 in 100,000 and there is no statistical evidence for the gender-biased occurrence of this syndrome.Abnormal regulation of growth deciding genes is the root cause of this disorder.

Researchers have stated that particular regions of chromosome 7 and 11contain group of genes that generally undergo genomic imprinting (Zeschnigk et al., 2008). At least one third of all cases of RSS root from methylation processes (Monk et al., 2002; Kotzot et al., 1995). RSS has been associated with changes in methylation genes involving H19 and IGF2, which are located on chromosome 11 (Saal, 2002; Duncan et al., 1990; Zeschnigk et al., 2008). Abnormalities occur on both the chromosomes 7 and 11 respectively:

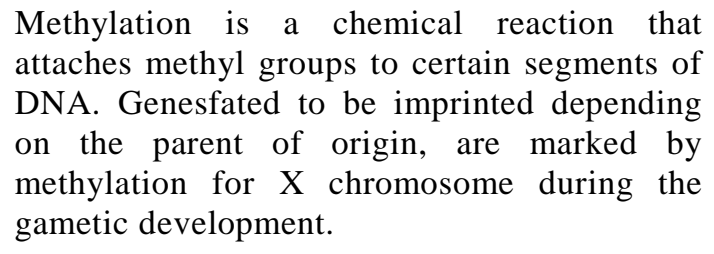

In RSS, $7-10 \%$ of instances are based oninheritanceof both copies of chromosome 7 maternally instead of one copy from each parent, which is termed as maternal Uniparental Disomy (UPD) (Preece et al., 1997; Henry et al., 1991). 


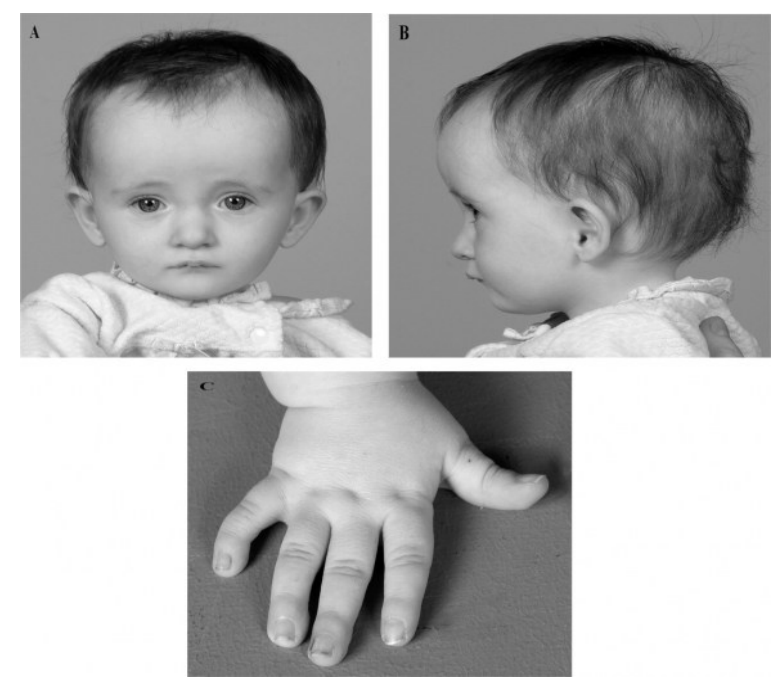

Fig. 4. Russell Silver Syndrome in a child (curvature of thumb and succumbed growth) (Source-http://manbironline.com/diseases/russell-silver.htm)

Maternal UPD causes people to have two active copies of maternally expressed imprinted genes rather than one active copy from the mother and one inactive copy from the father. These individuals lack a paternal copy of chromosome 7 and nullify the possibility of any copies of genes that are active only on the paternal copy. Such an imbalance in paternal and maternal genes on chromosome 7 shows the signs and symptoms of the disorder (Eggermann et al., 2009; Kotzot et al., 1995).

A significant proportion of people with Russell-Silver syndrome, have unidentified causes for the disorder and this highlights the modern research potentiality to look forchanges in chromosome other than 7 and 11 (Zeschnigk et al., 2008).

\subsection{Prader-Willi and Angelman Syndromes}

Prader-Willi Syndrome (PWS) and the Angelman Syndrome (AS) are associated with genomic imprinting on chromosome 15;q11-q13 and exhibited in terms of deficiencies in sexual development and growth and behavioral, hypotonia, hyperphagia and obesity, hypogonadism and developmental delay and mental problems including retardation (Magenis et al., 1987). Typically, AS patients display ataxia, tremulousness, sleep disorders, seizures, hyperactivity and transient escalation of happiness expressed in form of "Interspersed peaks of laughters". PWS and AS (autosomal dominant disorders) follow a strict disease inheritance pattern, i.e., only from one of the parent,thereby establishing the parent of origin theory
(Adams, 2008). De novobase deletion of the paternal or maternal chromosome 15(q11-q13) follows a rule of 3 megabase deletion for PWS and 4 megabase deletion for AS, highliting path modalities for approximately $70 \%$ of PWS and AS cases (Fig. 5). However, unlike RSS/BWS maternal UPD is lesser (25\%) in the case of PWS and paternal UPD is even more rare (4\%) for AS (Fig. 6) (Magenis et al., 1987; Viljoen and Ramesar, 1992).

Recent findings have shown that nearly $20 \%$ of the AS patients have had truncating mutations in UBE3A (encoding a ubiquitin protein ligase) which replaced the otherwise chromosomal deletion (Adams, 2008). Mapping of UBE3A to 15q11-q13, has validated its maternal specific expression in the human brain (Adams, 2008). Therefore, this finding helped to postulate the maternal-specific expression of UBE3Aand its abnormalities during brain development, as potential factors for AS.

The preferential loss of parental alleles associated with different phenotypes, coupled with the instances of UPD indicate the involvement of imprinted genes (i.e., paternally expressed gene (s) for PWS and maternally expressed gene(s) for AS) (Buiting et al., 1994). Broadly there are four imprinted, Paternally expressed genes for PWS (Adams, 2008; Viljoen and Ramesar, 1992):

- Small Nuclear Riboprotein-Associated Polypeptide N (SNRPN)

- Imprinted in Prader-Willi (IPW)

- Zinc Finger 127 (ZNF127)

- $\quad$ Necdin (NDN) 

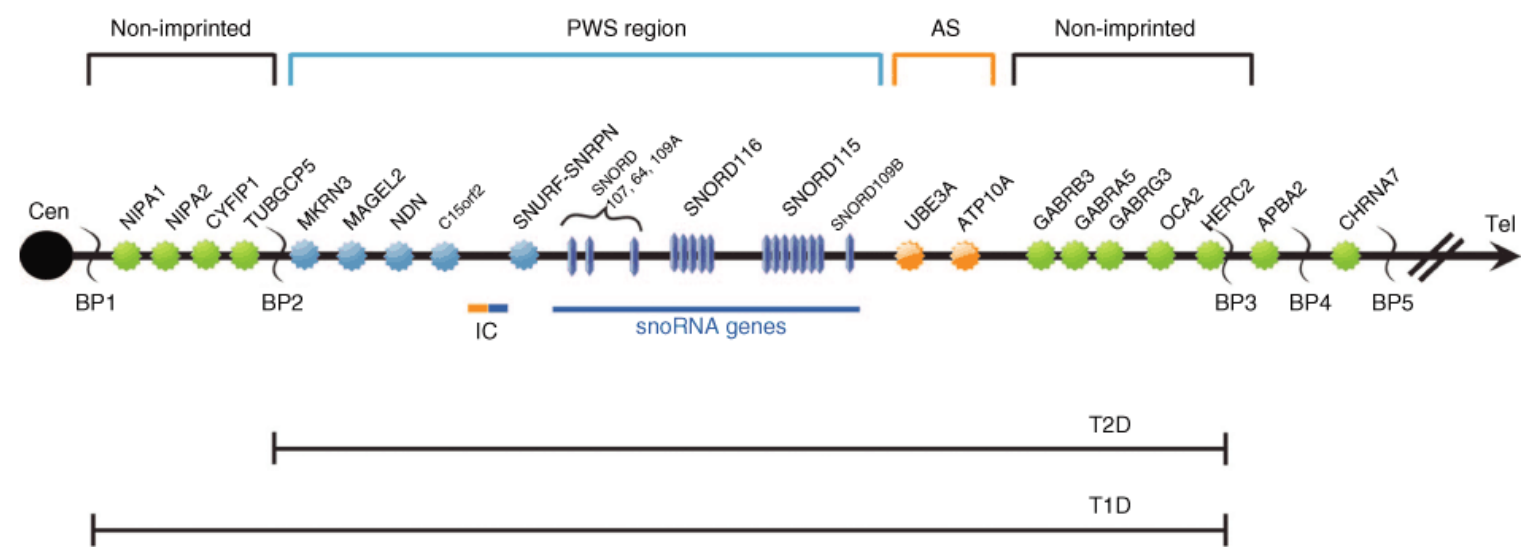

Fig. 5. Genetic Loci in Prader-Willi and Angelman Syndromes, (Sourcehttp://www.nature.com/gim/journal/v14/n1/abs/gim0b013e31822bead0a.html)

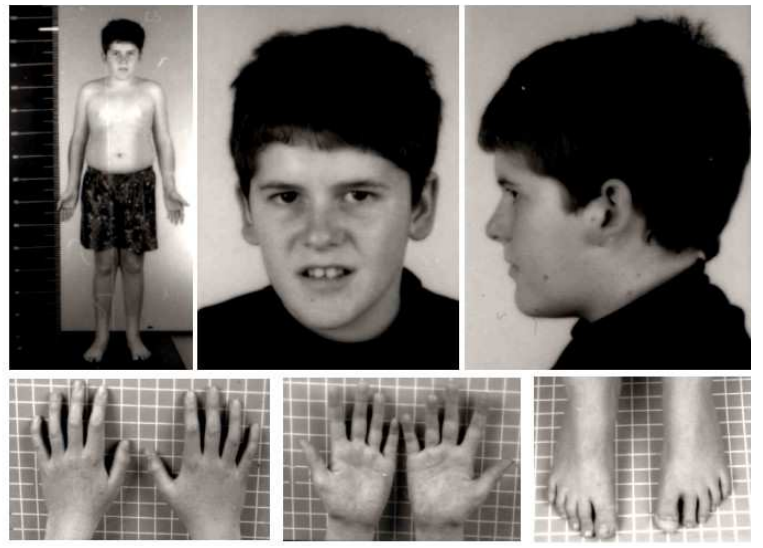

Fig. 6. 15 years aged boy showing Prader-Willi syndrome phenotype (absence of typical PWS facial features and presence of mild truncal obesity). (Source-Wikipedia.org)

Similarily, several paternally expressed transcripts may also be involved in PWS, like (Magenis et al., 1987; Viljoen and Ramesar, 1992):

- $\quad$ PAR1

- PAR5

- PAR-SN

Microdeletions of the SNRPN gene (pertaining to both parental complements of 15q11-q13) in a small percentage of PWS patients, has been accounted for the imprinting defects (Glenn et al., 1996). In context of the supression of paternal allele expression, these microdeletions alter the SNRPN promoter methylation and further silence a cluster of related genes.Apparently disruption of an imprinting centerinvolved in resetting the correct imprinting pattern during gametogenesis, is rendered by such events of microdeletions. In contrast, a small percentage of AS patients have similar microdeletions in the SNRPN gene, unlikely in the upstream sequences, which ultimately disturbs the rescue ability ofthe imprinting pattern (Adams, 2008):

- Paternal microdeletions-to progeny-do not develop AS

- Maternal transmission-to progeny-results in AS

Microdeletion in case of PWS and AS hold truein context of the IC hypothesis, also neccessiating a bipartite structure so that the uniqueness of minimally deleted regions responsible for PWS and AS, is maintained (Glenn et al., 1996).

\subsection{Neurological Disorder Model}

Some of the common neurological disorders have been explained with clues rooting from genetic imprinitng and associated abnormalities (BWS, 2003). 
Mental sickness has been concomitantly understood as an aspect of mentalism, defined by our grown ability to understand others action and behavior. Autistics, are people with limited abilities to conclude intentions or decipher the thoughtful false believes. Autistic Spectrum Disordersas such is a case of hypomentalism (BWS, 2003). Nevertheless, Psychotic Spectrum Disorders (PSDs) are getting newewer definitions with parameters like hypermentalism, paranoid schizophrenism. Symptomatical overinterpretation (either positively in erotomania (delusions that others are in love with you) or negatively in delusions of torture) substantiate these modern theories to greater extent. It has been seen that such patiennts start to believe strange false beliefs about themselves and others and generally they also display extreme mental situations, frequently preserved in supernatural delusions.

\section{CONCLUSION}

Extensive study of genetic imprinting and gene regulation in different hereditary disease models can be very efficient models for designing targeted drugs and also for understanding the molecular pathways and genetics of pathogenesis. It has been widely used in understanding a wide range of diseases and the search is still on to account for most of such epigenetically regulated diseases, so as to develop a consensus antidote again most common forms of such imprinting phenomenon and to a greater extent, the solution to the deadly cancer can be developed from our knowledge and applicability of genomic imprinting. Future possibilities of this field involve greater understanding of genomic imprinting in humans and identification of genetic locuses involving the zones of Imprinting Control Regions (ICRs) (BWS, 2003). This science has extensive utility in different domains of biomedical sciences and molecular biotechnology, that can give rise to extensive skills of engraving the pathogenicity of hereditary diseases.

\section{REFERENCES}

Adams, J., 2008. Imprinting and genetic disease: Angelman, Prader-Willi and Beckwith-Weidemann syndromes. Nature Educ., 1: 129-129.

Barlow, D.P., 1994. Imprinting: A gametes point of view. Trends. Genet., 10: 194-199. DOI: 10.1016/0168-9525(94)90255-0
Buiting, K., B. Dittrich and W.P. Robinson, 1994. Detection of aberrant DNA methylation in unique Prader-Willi syndrome patients and its diagnostic implications. Hum. Mol. Genet., 3: 893-895. DOI: $10.1093 / \mathrm{hmg} / 3.6 .893$

BWS, 2003. Beckwith-Wiedemann syndrome demonstrates a role for epigenetic control of normal development. Hum. Mol. Genet., 12: 61-68. DOI: 10.1093/hmg/ddg067

Cattanach, B.M., J. Barr and J. Jones, 1995. Use of Chromosome Rearrangements for Investigations into Imprinting in the Mouse. In: Genomic Imprinting: Causes and Consequences, Ohlsson, R., K. Hall and M. Ritzen (Eds.), Cambridge University Press, New York, ISBN-10: 0521472431, pp: 327-341.

Duncan, P.A., J.G. Hall, L.R. Shapiro and B.K. Vibert, 1990. Three-generation dominant transmission of the Silver-Russell syndrome. Am. J. Med. Genet., 35: 245-250. DOI: 10.1002/ajmg. 1320350220

Eggermann, T., D. Gonzalez, S. Spengler, M. ArslanKirchner and G. Binder et al., 2009. Broad clinical spectrum in Silver-Russell syndrome and consequences for genetic testing in growth retardation. Pediatrics, 123: e929-e931. DOI: 10.1542/peds.2008-3228, PMID: 19364767

Glenn, C.C., S. Saitoh, M.T. Jong, M.M. Filbrandt and U. Surti et al., 1996. Gene structure, DNA methylation and imprinted expression of the human SNRPN gene. Am. J. Hum. Genet., 58: 335-346. PMID: 8571960

Hall, J.G., 1990. Genomic imprinting: Review and relevance to human diseases. Am. J. Hum. Genet., 46: 857-873. PMID: 2187341

Henry, I., C. Bonaiti-Pellie, V. Chehensse, C. Beldjord and C. Schwartz et al., 1991. Uniparental paternal disomy in a genetic cancer-predisposing syndrome. Nature, 351: 665-667. DOI: 10.1038/351665a0

Kotzot, D., S. Schmitt, F. Bernasconi, W.P. Robinson and I.W. Lurie et al., 1995. Uniparental disomy 7 in Silver-Russell syndrome and primordial growth retardation. Hum. Mol. Genet., 4: 583-587. DOI: 10.1093/hmg/4.4.583

Ledbetter, D.H. and E. Engel, 1995. Uniparental disomy in humans: Development of an imprinting map and its implications for prenatal diagnosis. Hum. Mol. Genet., 4: 1757-1764. PMID: 8541876 
Magenis, R.E., M.G. Brown, D.A. Lacy, S. Budden and S. LaFranchi, 1987. Is Angelman syndrome an alternate result of $\operatorname{del}(15)(q 11 q 13)$ ? Am. J. Med. Genet., 28: 829-838. PMID: 3688021

Monk, D., L. Bentley, M. Hitchins, R.A. Myler and J. Clayton-Smith et al., 2002. Chromosome 7p disruptions in Silver Russell syndrome: Delineating an imprinted candidate gene region. Hum. Genet., 111: 376-387. DOI: 10.1007/s00439-002-0777-4

Monk, M. and A. Surani, 1990. Genomic Imprinting. Company of Biologists Ltd., Cambridge.

Munusamy, U., S.N.A. Abdullah, M.A. Aziz, H. Khazaai and L.M. Seong, 2013. Induced production of Îttocotrienol by co-suppression of tocopherol cyclase gene in vegetable crops. Am. J. Biochem. Biotechnol., 9: 355-364. DOI: 10.3844/ajbbsp.2013.355.364

Neumann, B., P. Kubicka and D.P. Barlow, 1995. Characteristics of imprinted genes. Nature Genet., 9: 12-13. DOI: $10.1038 / \mathrm{ng} 0195-12$

Nicholls, R.D., 1994. New insights reveal complex mechanisms involved in genomic imprinting. Am. J. Hum. Genet., 54: 733-740. PMID: 8178814

Ohlsoon, R., K. Hall and M. Ritzen, 1995. Genomic Imprinting-Causes and Consequences. 1st Edn., Cambridge University Press, New York, ISBN-10: 0521472431, pp: 374.

Preece, M.A., S.M. Price, V. Davies, L. Clough and P. Stanier et al., 1997. Maternal uniparental disomy 7 in Silver-Russell syndrome. J. Med. Genet., 34: 6-9. DOI: $10.1136 / j m g .34 .1 .6$
Reik, W., K.W. Brown and H. Schneid, 1995. Imprinting mutations in the Beckwith-Weidemann syndrome suggested by an altered imprinting pattern in the IGF2-H19 domain. Hum. Mol. Genet., 4: 23792385. DOI: $10.1093 / \mathrm{hmg} / 4.12 .2379$

Saal, H.M., 2002. Russell-Silver Syndrome. University of Washington. PMID: 20301499

Sapienza, C., 1994. Parental origin effects, genome imprinting and sex-ratio distortion: Double or nothing? Am. J. Hum. Genet., 55: 1073-1075. PMID: 7977364

Strain, L., J.P. Warner, T. Johnston and D.T. Bonthron, 1995. A human parthenogenetic chimera. Nature Genet., 11: 164-169. DOI: 10.1038/ng1095-164

Surani, M.A., 1995. Parthenogenesis in man. Nature Genet., 11: 111-113. DOI: 10.1038/ng1095-111

Tirumalai, P.S. and A.K. Bagchi, 2013. Magnum opus: Clustered regularly interspaced short palindromic repeats biology and prokaryotic gene silencing. Am. J. Immunol., 9: 110-115. DOI: 10.3844/ajisp.2013.110.115

Viljoen, D. and R. Ramesar, 1992. Evidence for paternal imprinting in familial Beckwith-Wiedemann syndrome. J. Med. Genet., 29: 221-225. DOI: 10.1136/jmg.29.4.221

Zeschnigk, M., B. Albrecht, K. Buiting, D. Kanber and T. Eggermann et al., 2008. IGF2/H19 hypomethylation in Silver-Russell syndrome and isolated hemihypoplasia. Eur. J. Hum. Genet., 16: 328-334. DOI: 10.1038/sj.ejhg.5201974 